\title{
Subgap states in disordered superconductors
}

\author{
M. A. Skvortsov and M. V. Feigel'man \\ L. D. Landau Institute for Theoretical Physics, 142432 Chernogolovka, Russia and \\ Moscow Institute of Physics and Technology, 141700 Moscow, Russia
}

(Dated: October 1, 2013)

\begin{abstract}
We revise the problem of the density of states in disordered superconductors. Randomness of local sample characteristics translates to the quenched spatial inhomogeneity of the spectral gap, smearing the BCS coherence peak. We show that various microscopic models of potential and magnetic disorder can be reduced to a universal phenomenological random order parameter model, whereas the details of the microscopic description are encoded in the correlation function of the order parameter fluctuations. The resulting form of the density of states is generally described by two parameters: the width $\Gamma$ measuring the broadening of the BCS peak, and the energy scale $\Gamma_{\text {tail }}$ which controls the exponential decay of the density of the subgap states. We refine the existing instanton approaches for determination of $\Gamma_{\text {tail }}$ and show that they appear as the limiting cases of a unified theory of optimal fluctuations in a nonlinear system. Application to various types of disorder is discussed.
\end{abstract}

PACS numbers: 74.78.-w, 74.20.-z, 74.81.-g

\section{INTRODUCTION}

Formation of the superconductive state is intimately related to the suppression of the quasiparticle density of states (DOS) in the vicinity of the Fermi energy. This effect is most pronounced for $s$-wave paring leading to a hard gap in the quasiparticle spectrum. If the timereversal invariance is not broken, the DOS follows the standard BCS expression,

$$
\rho_{\mathrm{BCS}}(E)=\rho_{0} \operatorname{Re} \frac{E}{\sqrt{E^{2}-\Delta^{2}}},
$$

where $\rho_{0}$ is the normal-metal DOS. Equation (10) applies both to clean and disordered systems [1, 2], indicating that thermodynamics of superconductors is insensitive to single-particle dynamics provided that a trajectory has its time-reversed counterpart needed to form a Cooper pair (Anderson theorem).

Breaking the time-reversal symmetry (e.g., by magnetic impurities [3], a supercurrent [4], a magnetic field in small superconducting grains/films [5]) lowers the critical temperature of the transition and smears the coherence peak (1). Various depairing scenarios are to a large extent equivalent [6] and can be described by a single dimensionless parameter

$$
\eta=\frac{1}{\tau_{\mathrm{dep}} \Delta_{0}}
$$

where $\tau_{\text {dep }}^{-1}$ is the depairing rate associated with a particular mechanism of time-reversal symmetry breaking, and $\Delta_{0}$ refers to the average value of the order parameter. According to the general analysis of Abrikosov and Gor'kov (AG) [3], the quasiparticle spectrum remains gapful for sufficiently weak pair breaking, $\eta<1$ (otherwise gapless superconductivity is expected). A new renormalized gap edge is located at

$$
E_{g}(\eta)=\left(1-\eta^{2 / 3}\right)^{3 / 2} \Delta_{0},
$$

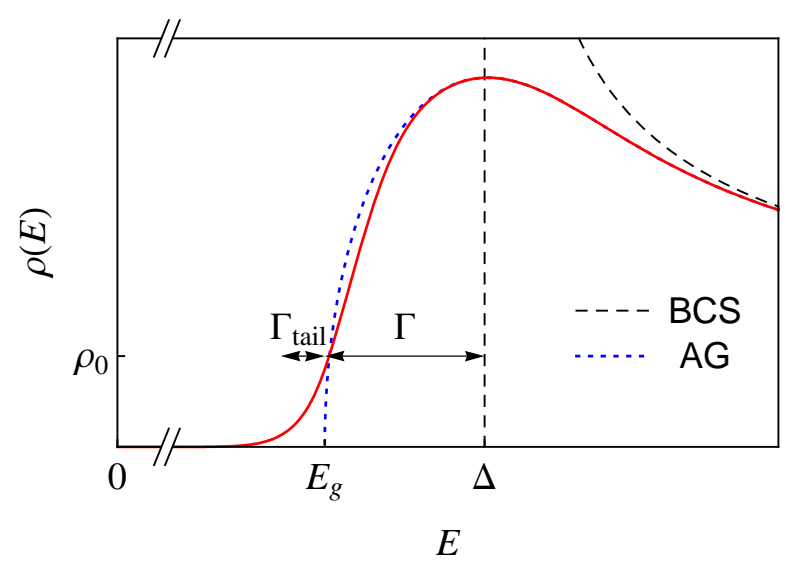

FIG. 1: Schematic view of the average DOS in a dirty superconductor (solid line). Broadening of the BCS peak (dashed line) is mainly described by the semiclassical approximation (dotted line), with the full DOS containing a significant tail of the subgap states.

with the DOS vanishing as $\rho(E) \propto\left(E-E_{g}\right)^{1 / 2}$, see dotted line in Fig. 1.

In the seminal paper back in 1971, Larkin and Ovchinnikov have recognized that the BCS-like form of the DOS may be smeared even if the time-reversal invariance is not broken [7]. They have considered a phenomenological model with a spatially varying Cooper-channel interaction constant, $\lambda(\mathbf{r})=\lambda_{0}+\delta \lambda(\mathbf{r})$, and have shown that short-scale disorder in $\lambda(\mathbf{r})$ has two effects on the DOS profile: First, at the mean-field level, it is equivalent to the AG model 3] with some effective deparing parameter $\eta$, therefore leading to the coherence peak smearing, but still with the hard gap at $E=E_{g}$. Second, this hard gap gets also smeared due to optimal fluctuations of the field $\lambda(\mathbf{r})$, leading to the Lifshitz-type [8, 9] tail of $\rho(E)$ in the subgap region, $E<E_{g}$.

The resulting form of the average DOS in a disor- 
dered superconductor is shown schematically in Fig. 1. Its main part is given by the AG theory (dotted line), with the coherent peak smearing controlled by an energy scale $\Gamma=\Delta_{0}-E_{g}$. This region corresponds to uniform configurations of the superconducting order parameter. On the contrary, the DOS tail at $E<E_{g}$ originates from the states localized in traps where the order parameter is lower than its average value $\Delta_{0}$. In this regime $\rho(E)$ strongly fluctuates in space, with the average DOS decaying typically as a stretched exponent at an energy scale $\Gamma_{\text {tail }}<\Gamma$ :

$$
\langle\rho(E)\rangle \propto \exp \left[-\left(\frac{E_{g}-E}{\Gamma_{\text {tail }}}\right)^{\zeta}\right] .
$$

Appearance of sufficiently deep traps is a rear event which is naturally identified with an instanton in the quasiclassical equations of superconductivity. Working in the dirty limit and studying optimal fluctuations in the Usadel equation [10], Larkin and Ovchinnikov [7] have calculated the average subgap DOS,

$$
\langle\rho(E)\rangle_{\mathrm{LO}} \propto \exp \left(-\alpha_{d}(\eta) \frac{\Delta_{0}^{2} \xi^{d}}{f(0)} \varepsilon^{(8-d) / 4}\right),
$$

which behaves as a stretched exponent of the dimensionless distance $\varepsilon$ from the gap edge,

$$
\varepsilon=\frac{E_{g}-E}{E_{g}},
$$

with the power $\zeta_{\mathrm{LO}}=(8-d) / 4$ dependent on the space dimensionality $d$ [11]. In Eq. (5), $\Delta_{0}$ is the average value of the order parameter, $\xi=\sqrt{D / 2 \Delta_{0}}$ is the superconducting coherence length ( $D$ is the diffusion coefficient), $f(0)$ is the zero Fourier harmonics of the correlation function of the order parameter fluctuations induced by quenched disorder in $\lambda(\mathbf{r})$ [see Eq. (17) below], and $\alpha_{d}(\eta)$ is a function of the dimensionless depairing parameter $\eta$ [see Eqs. (18) and (52) below].

The power of $\varepsilon$ in Eq. (5) can be easily understood within the optimal fluctuation approach. Near the AG threshold, at $E \rightarrow E_{g}$, the system is characterized by a diverging length scale $L_{E} \sim \xi \varepsilon^{-1 / 4}$ [7]. To have a quasiparticle state with an energy $E=(1-\varepsilon) E_{g}$ below the mean-field gap one has to locally reduce the order parameter by an amount of $\delta \Delta \sim \varepsilon \Delta_{0}$ in a volume specified by the length $L_{E}$. The price one has to pay for such an optimal fluctuation scales as $(\delta \Delta)^{2} L_{E}^{d} \sim \varepsilon^{2-d / 4}$, in accordance with the result (5).

Precisely the same model of a fluctuating Cooper constant [7] in a dirty superconductor has been reanalyzed thirty years later by Meyer and Simons [12] in the framework of the nonlinear $\sigma$ model approach. Using the instanton analysis of the $\sigma$ model, they have obtained a somewhat different optimal fluctuation leading to a different result for the tail of the subgap states:

$$
\langle\rho(E)\rangle_{\mathrm{MS}} \propto \exp \left(-\beta_{d}(\eta) g_{\xi} \varepsilon^{(6-d) / 4}\right),
$$

which is also a stretched exponent but with a different power $\zeta_{\mathrm{MS}}=(6-d) / 4$. Besides that, the instanton action of Meyer and Simons does not depend on the orderparameter correlation function $f(\mathbf{r})$. Instead, it contains some function $\beta_{d}(\eta)$ of the depairing parameter $\eta$ [see Eq. (43) below] and the dimensionless (in units of $e^{2} / h$ ) conductance $g_{\xi}$ of the region of size $\xi$ :

$$
g_{\xi}=4 \pi \nu D \xi^{d-2}=8 \pi \nu \Delta_{0} \xi^{d} .
$$

Appearance of the conductance $g_{\xi}$ in the exponent of Eq. (77) indicates that this expression cannot be obtained at the level of the saddle-point (Usadel) equation but requires the usage of the full nonlinear field theory.

Expression (7) for the density of subgap states has been obtained for a variety of disordered superconducting systems [12 16], where the semiclassical approximation predicts a square-root vanishing of the DOS, $\rho(E) \propto$ $\sqrt{E-E_{g}}$. In particular, it was observed in hybrid normal-metal - superconductor (NS) systems [14, 15], and in bulk superconductors with magnetic impurities [13, 16]. Mathematically it bears a close analogy with the Tracy-Widom distribution for the DOS tail in the Random matrix theory (RMT) [17], generalizing it from $d=0$ to an arbitrary dimensionality $d$. Based on these findings it is widely believed that Eq. (7) provides a universal description of the subgap DOS tail in disordered superconductors. However the discrepancy with the analysis of Larkin and Ovchinnikov existing at least for the model of the random Cooper channel constant still remains unresolved.

The purpose of this paper is to fill this gap by clarifying the origin of the two types of instantons discussed in Refs. 7 and 12 [leading to Eqs. (5) and (7)]. We will show that they correspond to different limits of a unique instanton solution realized for small and large $\varepsilon$, respectively. Hence, the Larkin-Ovchinnikov instanton can be continuously deformed into the Meyer-Simons instanton by changing the distance to the gap, $\varepsilon$. Such an unusual situation is a consequence of the nonlinearity of the Usadel equation. Therefore averaging over the random order parameter field $\Delta(\mathbf{r})$ produces a nonlinear term [8] which will compete with the intrinsic nonlinearity of the problem. This should be contrasted with the problem of fluctuation bound states in the Schrödinger equation with random potential [8, 9], where the only source of nonlinearity is due to averaging over disorder.

The paper is organized as follows. In Sec. [I] we introduce the random order parameter (ROP) model and derive its effective action in the large-scale limit. In Sec. III we analyze the instanton solutions with the broken replica symmetry and recover the results (5) and (7) in different limits. The summary and applications of the ROP model are discussed in Sec. [IV] Gap smearing in superconductors with magnetic impurities is reconsidered in Sec. V] We conclude with discussion of the results obtained in Sec. VI. Technical details are relegated to Appendix. 


\section{RANDOM ORDER PARAMETER MODEL}

\section{A. Model}

We start with the simplest example when the gap smearing results from quenched inhomogeneity in the pairing potential,

$$
\Delta(\mathbf{r})=\Delta_{0}+\Delta_{1}(\mathbf{r})
$$

which is assumed to be a real Gaussian random field specified by the correlation function

$$
\left\langle\Delta_{1}(\mathbf{r}) \Delta_{1}\left(\mathbf{r}^{\prime}\right)\right\rangle=f\left(\mathbf{r}-\mathbf{r}^{\prime}\right)
$$

The function $f(r)$ is supposed to be short-ranged, with the correlation length, $r_{c}$, being smaller than the superconducting coherence length [18]:

$$
r_{c}<\xi
$$

The superconductor is assumed to be in the dirty limit, $T_{c} \tau \ll 1$, where $\tau$ is the elastic scattering time.

The main simplification of this model, that will be referred to as the random order parameter (ROP) model, is that $\Delta(\mathbf{r})$ is considered as a given external field which should not be determined self-consistently.

The phenomenological ROP model universally emerges as an intermediate step in studying various types of disorder in the singlet case, when spin effects can be neglected [7, 12, 19] (a more general situation will be considered in Sec. V]. The function $f(\mathbf{r})$ in Eq. (10) then bears information on the original inhomogeneity in a particular microscopic model, see Sec. IVC.

\section{B. Sigma model}

The ROP model was treated by Larkin and Ovchinnikov [7] in terms of equations of motion (Usadel equation), and by Simons and co-authors [12, 13, 16] within the nonlinear $\sigma$-model formulation. Aiming to compare the two approaches, we choose to work in the functional language of the diffusive $\sigma$ model. To study the DOS in a field of a given $\Delta(\mathbf{r})$ at a particular energy $E$ one can use either its supersymmetric or replica version. We prefer to deal with the real-energy replica $\sigma$ model formulated in terms of the field $Q(\mathbf{r})$ acting in the direct product of the replica, Nambu and spin spaces (the latter is redundant in the singlet case considered but will be employed for the study of magnetic impurities in Sec. V] [13, 20 23].

Choosing the order parameter to be real, we write the $\sigma$-model action as

$$
S=\frac{\pi \nu}{4} \int d \mathbf{r} \operatorname{tr}\left[D(\nabla Q)^{2}+4\left(i E \tau_{3}-\Delta(\mathbf{r}) \tau_{1}\right) Q\right]
$$

where $\tau_{i}$ are Pauli matrices in the Nambu space.
Averaging over quenched disorder in $\Delta(\mathbf{r})$ with the help of Eqs. (9) and (10), we arrive at the action for the field $Q$ :

$$
S=S_{0}+S_{\mathrm{dis}}
$$

where

$$
\begin{aligned}
S_{0} & =\frac{\pi \nu}{4} \int d \mathbf{r} \operatorname{tr}\left[D(\nabla Q)^{2}+4\left(i E \tau_{3}-\Delta_{0} \tau_{1}\right) Q\right], \\
S_{\mathrm{dis}} & =-\frac{(\pi \nu)^{2}}{2} \int d \mathbf{r} d \mathbf{r}^{\prime} f\left(\mathbf{r}-\mathbf{r}^{\prime}\right) \operatorname{tr}\left[\tau_{1} Q(\mathbf{r})\right] \operatorname{tr}\left[\tau_{1} Q\left(\mathbf{r}^{\prime}\right)\right] .
\end{aligned}
$$

\section{Effective long-wavelength action}

The term $S_{\text {dis }}$ [Eq. (15)] contains an additional trace in the replica space and therefore does not contribute to the replica-diagonal saddle-point equation of motion. According to Larkin and Ovchinnikov 7], in order to see effects of disorder at the saddle-point level in the longwavelength limit (with momenta $q<q_{0}$ ), one has to average $S_{\text {dis }}$ over fast fluctuations of the field $Q$ (cooperons and diffusons). This procedure generates an effective depairing term [12, 23]

$$
S_{\eta}=-\frac{\pi \nu \Delta_{0} \eta}{4} \int d \mathbf{r} \operatorname{tr}\left(\tau_{3} Q\right)^{2}
$$

where the coefficient $\eta$ is expressed in terms of the Fourier transform of the order-parameter correlation function,

$$
f(\mathbf{q})=\left\langle\Delta_{1} \Delta_{1}\right\rangle_{\mathbf{q}}
$$

as

$$
\eta=\frac{2}{\Delta_{0}} \int \frac{f(\mathbf{q})}{D q^{2}} \frac{d^{d} \mathbf{q}}{(2 \pi)^{d}}
$$

In this derivation it was assumed that the regions of large momenta $\left(q>q_{0}\right)$ contributing to Eq. (18) and small momenta $\left(q<q_{0}\right)$ for which we derive an effective theory are well separated. This is true in 3D [7], marginally true in 2D [19] and wrong in 1D, see Sec. IVB for details.

Having eliminated short-range degrees of freedom we end up with an effective long-range $\left(r \gg r_{c}\right)$ action for the field $Q$ :

$$
S=S_{0}+S_{\eta}+S_{\text {dis }}
$$

where $S_{0}$ is given by Eq. (14), $S_{\eta}$ is given by Eq. (16), and $S_{\text {dis }}$ can be written in the local form:

$$
S_{\mathrm{dis}}=-\frac{(\pi \nu)^{2}}{2} f(0) \int d \mathbf{r}\left[\operatorname{tr} \tau_{1} Q(\mathbf{r})\right]^{2} .
$$

At this stage we may trace the difference between the approaches of Refs. 77] and [12]. In order to reproduce the analysis of Larkin and Ovchinnikov [7] one has to decouple the term $S_{\text {dis }}$ [Eq. (20)] with the Gaussian whitenoise order parameter field $\Delta_{1}(\mathbf{r})$ and treat the resulting 
problem in the saddle-point approximation assuming the solution is replica symmetric. As we will see in Sec.IIID, in terms of the $Q$-only action (19) this corresponds to instanton solutions with infinitesimally small replica symmetry breaking. On the other hand, Meyer and Simons [12] did not use the saddle-point approximation but completely neglected the term $S_{\text {dis }}$ which accounts for longrange fluctuations of the order-parameter field. Their instanton solution originating from the nonlinearity of the underlying field theory has a nontrivial replica structure discussed in Sec. III C. Below we will analyze the action (19) and clarify the validity of approximations employed in Refs. [7] and [12].

\section{OPTIMAL FLUCTUATIONS IN A NON-LINEAR SYSTEM}

\section{A. Saddle-point equations}

Here we analyze the saddle points of the action (19) which have the replica-diagonal form:

$$
\left(Q_{0}\right)^{a b}=\delta_{a b}\left[\tau_{3} \cos \theta^{a}+\tau_{1} \sin \theta^{a}\right],
$$

where Latin indices refer to the replica space, and the spectral angle $\theta^{a}(E)$ depends on the energy considered.

The simplest is the replica-symmetric saddle point, with $\theta^{a}=\theta_{0}$ for all $a=1, \ldots, n$. For a replica-symmetric solution, the actions $S_{0}$ and $S_{\eta}$ are proportional to the number of replicas, $n$, whereas the action $S_{\text {dis }}$ is proportional to $n^{2}$ and does not contribute to the saddle-point (Usadel) equation in the replica limit $n \rightarrow 0$. Then the saddle-point equation for a uniform $Q_{0}$ immediately reproduces the $\mathrm{AG}$ equation for the spectral angle in the model of magnetic impurities [3] :

$$
i E \sin \theta_{0}+\Delta_{0} \cos \theta_{0}-\Delta_{0} \eta \cos \theta_{0} \sin \theta_{0}=0 .
$$

The corresponding DOS, $\rho(E)=\rho_{0} \operatorname{Re} \cos \theta_{0}$, characterized by the hard gap at $E_{g}[\mathrm{Eq}$. (3)] is shown by the dotted line in Fig. 1 .

The subgap states are associated with localized saddlepoint solutions with broken replica symmetry [12]. To study them it is convenient to write [13, 15]

$$
\theta^{a}=\pi / 2+i \psi^{a}
$$

The replica-symmetric mean-field solution, $\psi^{a}=\psi_{0}$, is real for $E<E_{g}$, and appearance of a finite DOS is related to configurations with complex $\psi$.

The set of the saddle-point equations for the action (19) in terms of the angle $\psi$ takes the form:

$$
-\xi^{2} \nabla^{2} \psi^{a}+F\left(\psi^{a}\right)+\kappa \sinh \psi^{a} \sum_{b} \cosh \psi^{b}=0
$$

where $\xi$ is the coherence length, $\xi^{2}=D / 2 \Delta_{0}$, the parameter $\kappa$ is related to the low-momentum correlator of the order parameter fluctuations:

$$
\kappa=\frac{4 \pi \nu f(0)}{\Delta_{0}}=\frac{g_{\xi}}{2} \frac{f(0)}{\Delta_{0}^{2} \xi^{d}}
$$

and the function $F(\psi)$ is given by

$$
F(\psi)=-\frac{E}{\Delta_{0}} \cosh \psi+\sinh \psi-\eta \sinh \psi \cosh \psi
$$

Equation $F(\psi)=0$ is equivalent to the AG equation (22).

Behavior of the function $F(\psi)$ for real arguments depends on the relation between $E$ and $E_{g}$. Below the gap $\left(E<E_{g}\right)$, equation $F(\psi)=0$ has two solutions: $\psi_{0}$ (AG solution) and $\psi^{\prime}>\psi_{0}$. They merge at $E=E_{g}$, where an analytic solution is possible, yielding

$$
\cosh \psi_{g}=\eta^{-1 / 3}
$$

Above the gap $\left(E>E_{g}\right)$, equation $F(\psi)=0$ has no real solutions. For small deviation from the gap, $E \rightarrow E_{g}$, the function $F(\psi)$ can be expanded near the AG solution as

$$
F(\psi) \approx \Omega\left(\psi-\psi_{0}\right)-\rho\left(\psi-\psi_{0}\right)^{2},
$$

with the dimensionless parameters

$$
\Omega=\left(1-\eta^{2 / 3}\right) \sqrt{6 \varepsilon}, \quad \rho=\frac{3}{2} \eta^{1 / 3} \sqrt{1-\eta^{2 / 3}},
$$

where $\varepsilon$ is defined in Eq. (6).

\section{B. Instantons with broken replica symmetry}

Now we concentrate on solutions of Eqs. (24) with the simplest nontrivial structure in the replica space [12]:

$$
\psi^{a}(\mathbf{r})= \begin{cases}\psi_{1}(\mathbf{r}), & a=1 \\ \psi_{2}(\mathbf{r}), & a=2, \ldots, n\end{cases}
$$

Such a solution is characterized by two functions, $\psi_{1}(\mathbf{r})$ and $\psi_{2}(\mathbf{r})$, which satisfy the system of two coupled nonlinear equations (24), where

$$
\sum_{b} \cosh \psi^{b}=\cosh \psi_{1}-\cosh \psi_{2}
$$

in the replica limit $(n \rightarrow 0)$.

The system (24) simplifies in the vicinity of the gap edge, $E \rightarrow E_{g}$, where variations of $\psi_{1}(\mathbf{r})$ and $\psi_{2}(\mathbf{r})$ are small and the replica-mixing term may be linearized. To write the resulting equations in a dimensionless form, we measure distance in units of the divergent length scale [7]

$$
L_{E}=\frac{\xi}{\sqrt{\Omega}} \sim \xi\left(\frac{E_{g}}{E_{g}-E}\right)^{1 / 4}
$$

and write

$$
\psi_{1,2}(\mathbf{r})=\psi_{0}+(\Omega / \rho) \phi_{1,2}(\mathbf{r})
$$


As a result, we arrive at the following system:

$$
\begin{aligned}
& -\nabla^{2} \phi_{1}+\phi_{1}-\phi_{1}^{2}=K(\varepsilon)\left(\phi_{2}-\phi_{1}\right), \\
& -\nabla^{2} \phi_{2}+\phi_{2}-\phi_{2}^{2}=K(\varepsilon)\left(\phi_{2}-\phi_{1}\right) .
\end{aligned}
$$

The replica mixing is controlled by the the single dimensionless parameter $K(\varepsilon)$ :

$$
K(\varepsilon)=\sqrt{\frac{\varepsilon_{*}}{\varepsilon}},
$$

where the energy scale $\varepsilon_{*}$ given by

$$
\varepsilon_{*}=\frac{\kappa^{2}}{6 \eta^{4 / 3}}=\frac{g_{\xi}^{2}}{24 \eta^{4 / 3}}\left(\frac{f(0)}{\Delta_{0}^{2} \xi^{d}}\right)^{2} .
$$

Sufficiently close to the gap edge, at $\varepsilon \lesssim \varepsilon_{*}$, the parameter $K(\varepsilon)$ is large and equations for $\phi_{1}(\mathbf{r})$ and $\phi_{2}(\mathbf{r})$ are strongly coupled. Small values of $K(\varepsilon)$ can be realized only for large deviations from the gap, at $\varepsilon \gtrsim \varepsilon_{*}$.

With the exponential accuracy the subgap DOS is determined by the instanton action:

$$
\langle\rho(E)\rangle \propto \exp \left(-\gamma_{d}(\eta) g_{\xi} \varepsilon^{(6-d) / 4} S_{0}[K(\varepsilon)]\right),
$$

where $g_{\xi}$ given by Eq. (8) is the dimensionless conductance of the region of size $\xi$,

$$
\gamma_{d}(\eta)=\frac{4}{3} 6^{(2-d) / 4} \frac{\left(1-\eta^{2 / 3}\right)^{2-d / 2}}{\eta^{2 / 3}},
$$

and $S_{0}(K)$ is the dimensionless instanton action:

$$
S_{0}(K)=\frac{1}{6} \int\left(\phi_{2}^{3}-\phi_{1}^{3}\right) d \mathbf{r} .
$$

Note that the energy dependence of the average DOS (37) comes both from the factor $\varepsilon^{(6-d) / 4}$ and the energy dependence of the parameter $K(\varepsilon)$. Below we will analyze solutions of Eqs. (34) in the limiting cases of small and large values of $K$ and identify them with the MeyerSimons and Larkin-Ovchinnikov instantons, respectively.

\section{Instanton in the limit $K \rightarrow 0$}

In the limit $K \rightarrow 0$, Eqs. (34) decouple yielding a single equation

$$
-\nabla^{2} \phi+\phi-\phi^{2}=0
$$

both for $\phi_{1}(\mathbf{r})$ and $\phi_{2}(\mathbf{r})$. This equation has three solutions: two constant solutions, $\phi(\mathbf{r})=0$ (corresponding to the AG solution) and $\phi(\mathbf{r})=1$, and a spherically symmetric bounce $\phi_{\text {inst }}^{(d)}(r)$ vanishing for $r \rightarrow \infty$. The bounce solution of Eq. (40) is known explicitly in the 1D geometry:

$$
\varphi_{\text {inst }}^{(1)}(r)=\frac{3}{2 \cosh ^{2}(r / 2)},
$$

and can be obtained numerically for other dimensionalities. The instanton action is determined by the number

$$
s_{d} \equiv \frac{1}{6} \int\left[\varphi_{\text {inst }}^{(d)}(r)\right]^{3} d \mathbf{r}= \begin{cases}6 / 5, & d=1, \\ 7.75, & d=2, \\ 43.7, & d=3 .\end{cases}
$$

To minimize the action (39) we take the trivial AG solution $\phi_{1}(\mathbf{r})=0$ for the first replica and choose a bounce solution, $\phi_{2}(\mathbf{r})=\varphi_{\text {inst }}^{(d)}(r)$, for the other replicas. Hence, $S_{0}(0)=s_{d}$ and Eq. (37) reproduces the result (17) of Refs. 12 and 13 with

$$
\beta_{d}(\eta)=s_{d} \gamma_{d}(\eta)
$$

\section{Instanton for $K \rightarrow \infty$, optimal fluctuation, and dimensional reduction}

In the limit $K \rightarrow \infty$, the last terms in Eqs. (34) render $\phi_{1}(\mathbf{r})$ and $\phi_{2}(\mathbf{r})$ nearly equal. So we may expand their difference in powers of $K^{-1}$ and write

$$
\phi_{1}(\mathbf{r})=\phi(\mathbf{r}), \quad \phi_{2}(\mathbf{r})=\phi(\mathbf{r})+K^{-1} \chi(\mathbf{r})+\ldots
$$

Substituting this expansion into Eqs. (34) we get

$$
\begin{gathered}
-\nabla^{2} \phi+\phi-\phi^{2}=\chi, \\
-\nabla^{2} \chi+\chi-2 \phi \chi=0 .
\end{gathered}
$$

Now excluding $\chi(\mathbf{r})$, we come to the fourth-order differential equation for the function $\phi(\mathbf{r})$ :

$$
\left[-\nabla^{2}+1-2 \phi\right]\left[-\nabla^{2} \phi+\phi-\phi^{2}\right]=0 .
$$

Equation (46) naturally appears in the study of optimal fluctuations in a nonlinear equation

$$
-\nabla^{2} \phi+F(\phi)=h(\mathbf{r}),
$$

where $F(\phi)=\phi-\phi^{2}$ and $h(\mathbf{r})$ is a Gaussian $\delta$-correlated random field [7]. Optimal fluctuation arguments [8, 9] lead to the minimization of the functional $\int h^{2} d \mathbf{r}=$ $\int\left[-\nabla^{2} \phi+F(\phi)\right]^{2} d \mathbf{r}$, and hence to the saddle-point equation

$$
\left[-\nabla^{2}+F^{\prime}(\phi)\right]\left[-\nabla^{2} \phi+F(\phi)\right]=0,
$$

coinciding with Eq. (46). Thus we see that in the replica formalism the role of the random field $h(\mathbf{r})$ is played by the mismatch of solutions for different replicas: $\chi(\mathbf{r}) \propto$ $\phi_{2}(\mathbf{r})-\phi_{1}(\mathbf{r})$.

Note that those $\phi(\mathbf{r})$ solving Eq. (40) also solve Eq. (46). However none of them correspond to an optimal fluctuation since they have $h=0$ and do not lead to a finite DOS. Therefore one has to look for another solution of Eq. (46). Quite surprisingly, for the spherically 
symmetric solutions $\phi(r)$, there exists an identity valid for an arbitrary function $F(\phi)$ and arbitrary $d$ [24]:

$$
\begin{aligned}
& {\left[-\Delta_{\mathrm{rad}}^{(d)}+F^{\prime}(\phi)\right]\left[-\Delta_{\mathrm{rad}}^{(d)} \phi+F(\phi)\right]} \\
& \quad=\left[-\Delta_{\mathrm{rad}}^{(d+2)}+F^{\prime}(\phi)\right]\left[-\Delta_{\mathrm{rad}}^{(d-2)} \phi+F(\phi)\right]
\end{aligned}
$$

where $\Delta_{\text {rad }}^{(d)}$ is the radial part of the Laplace operator in $d$ dimensions:

$$
\Delta_{\mathrm{rad}}^{(d)}=\frac{1}{r^{d-1}} \frac{\partial}{\partial r} r^{d-1} \frac{\partial}{\partial r}
$$

Thus there is a kind of a dimensional reduction: a nontrivial optimal fluctuation in $d$ dimensions solving Eq. (46) is just the bounce solution of Eq. (40) in $d-2$ dimensions. Note that a somewhat similar dimensional reduction has been obtained in Ref. 25 for the critical behavior of spin systems in a random magnetic field.

The instanton action (39) is given by

$$
S_{0}(K)=\frac{1}{2 K} \int \phi^{2} \chi d \mathbf{r}=\frac{1}{2 K} \int \chi^{2} d \mathbf{r},
$$

where the last relation follows from Eqs. (45). In the optimal fluctuation language, $\exp [-S(K)]$ is just the probability density for the Gaussian random field $\chi(\mathbf{r})$. Substituting Eq. (51) into the general expression (37), we arrive at the result (5), where

$$
\alpha_{d}(\eta)=16 \cdot 6^{-d / 4} c_{d}\left(1-\eta^{2 / 3}\right)^{2-d / 2},
$$

and $c_{d}=\lim _{K \rightarrow \infty} K S_{0}(K)$ is the $d$-dependent constant:

$$
c_{d}=2 \int\left(\frac{\partial \varphi_{\mathrm{inst}}^{(d-2)}(r)}{\partial r}\right)^{2} \frac{d \mathbf{r}}{r^{2}}= \begin{cases}0.266, & d=1 \\ 2.09, & d=2 \\ 24 \pi / 5, & d=3\end{cases}
$$

In the $3 \mathrm{D}$ case and at $\eta \ll 1$, Eq. (5) coincides with the result of Ref. 7 (where only this limit was considered). Thus our instantons in the limit $K \rightarrow \infty$ directly correspond to the optimal fluctuations of Larkin and Ovchinnikov, and the dimensional reduction (49) explains why did they manage to find an explicit analytic expression for the optimal fluctuation in the 3D case: $\phi^{(3)}(r)=\varphi_{\text {inst }}^{(1)}(r)$, with the latter given by Eq. (41).

\section{E. Intermediate values of $K$}

Two types of instantons analyzed above continuously interpolate between each other with variation of $K$. As an example we show in Fig. 2 numerical solutions of 1D equations (34) for some intermediate values of $K$. For small but finite $K$, solutions $\phi_{1}(r)$ and $\phi_{2}(r)$ start to deviate from 0 and $\varphi_{\text {inst }}^{(d)}(r)$, respectively, moving towards each other. Already at $K=1$ they are close, approaching the asymptote $\varphi_{\text {inst }}^{(d-2)}(r)$ at $K \rightarrow \infty$.

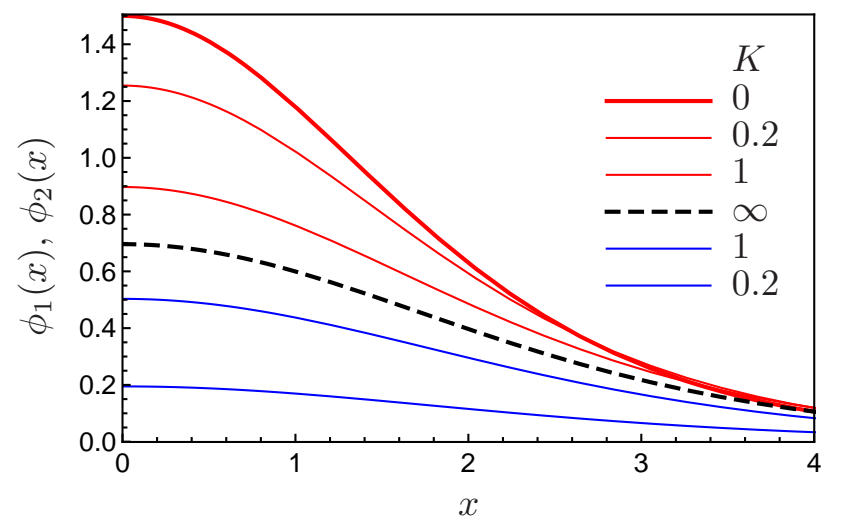

FIG. 2: Numerical solutions of Eqs. (34) in the 1D geometry $(d=1)$ for various values of the replica-mixing parameter $K$. For $K=0, \phi_{1}(x)=0$ and $\phi_{2}(x)=\varphi_{\text {inst }}^{(1)}(x)$ (upper thick curve). Solutions for intermediate values $(K=0.2$ and $K=1$ ) are shown by the thin solid lines. In the limit $K \rightarrow$ $\infty, \phi_{1}(x)$ and $\phi_{2}(x)$ approach the asymptote $\varphi_{\text {inst }}^{(-1)}(x)$ (thick dashed line).

Hence, with increasing the deviation from the gap edge into the classically forbidden region, one gradually crosses over from the Larkin-Ovchinnikov to the MeyerSimons instanton. The crossover takes place at the dimensionless energy $\varepsilon_{*}$ given by Eq. (36). Equivalently the latter may be estimated just by equating the results (5) and (7).

\section{RANDOM ORDER PARAMETER MODEL: SUMMARY AND APPLICATIONS}

Having established the replica structure of the instantons in the ROP model we now comment on the limits of validity of the above analysis and consider various applications of the ROP model in a more general context.

\section{A. Validity of the instanton analysis}

Our analysis was based on two implicit assumptions: (i) large instanton action allowing to use the saddle-point approximation, and (ii) small deviation from the gap edge, $\varepsilon \ll 1$, allowing to expand Eq. (26) and get the universal system (34). Once we know the resulting expressions (5) and (7) for the DOS tails (at $\varepsilon \ll \varepsilon_{*}$ and $\varepsilon \gg \varepsilon_{*}$, respectively), we can verify these assumptions a posteriori. Depending on the relations between the parameters of the ROP model one can identify the following regimes:

I. $f(0) / \Delta_{0}^{2} \xi^{d}<\left(\eta^{2 / 3} / g_{\xi}\right)^{(8-d) /(6-d)}$.

The tail is described by Eq. (7) for all $\varepsilon$. The Larkin-Ovchinnikov tail does not exist since the corresponding action is smaller than 1. 
IIA. $\left(\eta^{2 / 3} / g_{\xi}\right)^{(8-d) /(6-d)}<f(0) / \Delta_{0}^{2} \xi^{d}<\eta^{2 / 3} / g_{\xi}$.

The main part of the tail (for small $\varepsilon<\varepsilon_{*}$ ) follows the Larkin-Ovchinnikov result (5). The tail (7) exists at large $\varepsilon>\varepsilon_{*}$ where it is extremely small.

IIB. $\eta^{2 / 3} / g_{\xi}<f(0) / \Delta_{0}^{2} \xi^{d}$.

The tail follows the Larkin-Ovchinnikov result (5) for all $\varepsilon$. The tail (7) does not exist.

In each of the regimes only one type of the instantons is important. The other one either does not exist or is unobservable.

\section{B. Role of dimensionality}

Larkin and Ovchinnikov approach 77] to the ROP model is based on the idea of separation of scales: shortscale fluctuations of $\Delta(\mathbf{r})$ produce an effective depairing $\eta$ leading to the formation of the AG-like hard gap, whereas long-scale fluctuations are responsible for the gap smearing. Due to the presence of the diffusive pole in the integrand in Eq. (18) for $\eta$, the possibility of such separation depends on the dimensionality of the problem.

In 3D, the integral in Eq. (18) is determined by large momenta, $q \sim r_{c}^{-1}$, where $r_{c}$ is the correlation length of the fluctuating field $\Delta_{1}(\mathbf{r})$, leading to the estimate

$$
\eta_{3 \mathrm{D}}=\frac{1}{2 \pi^{2} \Delta_{0}^{2} \xi^{2}} \int_{0}^{\infty} f(q) d q \sim \frac{f(0)}{2 \pi^{2} \Delta_{0}^{2} \xi^{2} r_{c}} .
$$

The long-wavelength theory (19) is then applicable already for scales $r \gtrsim r_{c}$.

The 2D case is marginal since the integral in Eq. (18) is logarithmic. Its upper cutoff is again given by $r_{c}^{-1}$, whereas the lower limit must be determined with care. According to Ref. [19], with logarithmic accuracy an appropriate cutoff is established by replacing $D q^{2} \mapsto D q^{2}+$ $D / L_{E}^{2}$, where the length $L_{E}$ is defined in Eq. (32). As a result, the depairing factor becomes energy-dependent:

$$
\eta_{2 \mathrm{D}}(E)=\frac{1}{2 \pi} \frac{f(0)}{\Delta_{0}^{2} \xi^{2}} \ln \frac{\min \left(L_{E}, L_{g}\right)}{r_{c}},
$$

where we had to introduce an infrared length scale $L_{g}$ in order to regularize the otherwise divergent $\eta_{2 \mathrm{D}}\left(E \rightarrow E_{g}\right)$. Its appearance is related to the breakdown of the meanfield approximation in the narrow region $\left|E-E_{g}\right| \lesssim \Gamma_{\text {tail }}$, where proliferation of instantons generates a finite correlation length $L_{g} \sim \xi\left(E_{g} / \Gamma_{\text {tail }}\right)^{1 / 4}$. Here one should use the Meyer-Simons (Larkin-Ovchinnikov) expression for $\Gamma_{\text {tail }}$ provided the regime I (II) is realized [see discussion in Sec. IVA.

In $1 \mathrm{D}$, the integral (18) is divergent in the infrared, indicating that analytical treatment based on the idea of scale separation is not possible, and $\Gamma_{\text {tail }} \approx \Gamma$.

\section{Applications of the ROP model}

\section{Random coupling constant model}

This is the model initially considered by Larkin and Ovchinnikov [7]. It can be reduced to the ROP model in the following way. A fluctuating Cooper constant introduces quenched fluctuations in the order parameter field which should be determined from the self-consistency equation. The latter can be written in the Matsubara representation and solved perturbatively. In the linear order one gets [7]

$$
\lambda_{0}^{-1} \Delta_{1}(\mathbf{q})+\delta \lambda^{-1}(\mathbf{q}) \Delta_{0}=\pi T \sum_{\epsilon} \frac{\partial F(\epsilon, \mathbf{q})}{\partial \Delta(\mathbf{q})} \Delta_{1}(\mathbf{q}),
$$

where $F(\epsilon, \mathbf{q})$ is the Fourier transform of the quasiclassical Gor'kov function $F(\epsilon, \mathbf{r})=\sin \theta(\epsilon, \mathbf{r})$, and its derivative with respect to $\Delta_{1}$ can be obtained from the Usadel equation. Solving for $\Delta_{1}(\mathbf{q})$ we arrive at the linear relation

$$
\Delta_{1}(\mathbf{q})=-\Delta_{0} L_{0}(q) \delta \lambda^{-1}(\mathbf{q}),
$$

where $L_{0}(q)$ is the static propagator of superconducting fluctuations in the BCS theory:

$$
L_{0}^{-1}(q)=\pi T \sum_{\epsilon} \frac{\Delta_{0}^{2}+\mathfrak{E}(\epsilon) D q^{2} / 2}{\mathfrak{E}^{2}(\epsilon)\left[\mathfrak{E}(\epsilon)+D q^{2} / 2\right]},
$$

and

$$
\mathfrak{E}(\epsilon)=\sqrt{\epsilon^{2}+\Delta_{0}^{2}}
$$

At zero temperature integration over Matsubara energies $\epsilon$ can be performed analytically [7, 12, leading to:

$$
\begin{aligned}
L_{0}(q)=\frac{2 \tilde{q}^{2}}{\pi-4 \sqrt{1-\tilde{q}^{4}} \arctan \sqrt{\frac{1-\tilde{q}^{2}}{1+\tilde{q}^{2}}}} & \\
= & \begin{cases}1-\pi \tilde{q}^{2} / 4+\ldots, & \tilde{q} \ll 1 ; \\
1 / \ln \tilde{q}^{2}+\ldots, & \tilde{q} \gg 1 ;\end{cases}
\end{aligned}
$$

where $\tilde{q}=q \xi$.

Thus we end up with the ROP model specified by the correlation function

$$
f(q) \equiv\left\langle\Delta_{1} \Delta_{1}\right\rangle_{\mathbf{q}}=\Delta_{0}^{2} L^{2}(q)\left\langle\delta \lambda^{-1} \delta \lambda^{-1}\right\rangle_{\mathbf{q}} .
$$

The random coupling constant model can be mapped onto the ROP model as long as fluctuations of $\lambda(\mathbf{r})$ are weak (the resulting depairing parameter $\eta \ll 1$ ). Otherwise it is not sufficient to use the first-order perturbation theory in Eq. (56). 


\section{Gap fluctuations in NS systems}

The simplest setup where disorder leads to formation of the subgap states is the model of the NS junction [14, 15]. Here superconductive correlations are induced into the normal metal due to the proximity effect, opening the (mini)gap in the excitation spectrum [26]. In a long diffusive junction (with size $L \gg \xi$ ) a minigap is of the order of the Thouless energy: $E_{g} \sim E_{\mathrm{Th}}=D / L^{2}$ [27, 28]. In the normal part of the junction the order parameter is absent and the only source of disorder is due to random position of potential impurities. These mesoscopic fluctuations are known to be responsible for various sample-to-sample fluctuations such as universal conductance fluctuations 29], mesoscopic fluctuations of the Josephson current [30, 31], etc. [32].

The DOS tail due to mesoscopic fluctuations in NS hybrid systems [14, 15] follows the result (7). This sheds light on the physical origin of the instanton of the action $S_{0}+S_{\eta}$ discussed in Sec. IIIC it describes DOS smearing due to mesoscopic fluctuations of the quasiparticle response to a constant (for this particular problem, zero) order parameter. In other words, randomness of impurities' positions translates into fluctuations of the quasiparticle Green function $Q(\mathbf{r})$.

\section{Mesoscopic fluctuations of the order parameter}

In disordered superconductors, mesoscopic fluctuations lead to fluctuations of the order parameter [19, 33]. The latter are generated by fluctuations of $Q(\mathbf{r})$ if one takes the self-consistency equation into account.

In the 2D case and in the presence of the Coulomb interaction, the order parameter correlation function was calculated in Ref. [19]. The correlation length of these fluctuations is of the order of the zero-temperature coherence length, $r_{c} \sim \xi(0)$. Their magnitude at $T=0$ and $q=0$ are given by

$$
\frac{f_{2 \mathrm{D}}^{\mathrm{MF}+\text { Coulomb }}(0)}{\Delta_{0}^{2} \xi^{2}}=\frac{2 \pi}{g\left(g-g_{c}\right)},
$$

where $g_{c}=\ln ^{2}\left(\hbar / T_{c 0} \tau\right) / 2 \pi$ is the critical conductance for the fermionic mechanism of the superconductivity suppression [34] $\left(T_{c 0}\right.$ is the transition temperature in the clean system and $\tau$ is the elastic scattering time).

Evaluating the depairing parameter from Eq. (55), we find that the regime IIB is always realized [see Sec. IVA]. Therefore gap smearing in homogeneously disordered superconducting films is always described by the LarkinOvchinnikov mechanism leading to Eq. (5), and there is no room for the Meyer-Simons instanton [19].

For completeness we present here the general expression for the correlation function of the order parameter due to mesoscopic fluctuations at arbitrary space dimen- sionality $d$ but in the absence of the Coulomb effects:

$$
f^{\mathrm{MF}}(q)=\frac{L_{0}^{2}(q) \Delta_{0}^{2} T^{2}}{\nu^{2}} \sum_{\epsilon, \epsilon^{\prime}} \int \frac{\Pi_{\epsilon \epsilon^{\prime}}(k) \Pi_{\epsilon \epsilon^{\prime}}(k-q)}{\mathfrak{E}(\epsilon) \mathfrak{E}\left(\epsilon^{\prime}\right)} \frac{d^{d} \mathbf{k}}{(2 \pi)^{d}},
$$

where $\mathfrak{E}(\epsilon)$ is defined in Eq. (59), and $\Pi_{\epsilon \epsilon^{\prime}}(k)=\left[D k^{2}+\right.$ $\left.\mathfrak{E}(\epsilon)+\mathfrak{E}\left(\epsilon^{\prime}\right)\right]^{-1}$ is the diffusion propagator on top of the BCS state. In particular, at $T=0$ and $q=0$ one gets

$$
\frac{f^{\mathrm{MF}}(0)}{\Delta_{0}^{2} \xi^{d}} \sim \frac{1}{g_{\xi}^{2}}
$$

Since mesoscopic fluctuations are inevitably present in any disordered system, Eq. (64) is the lower bound for the order parameter fluctuations in disordered superconductors.

\section{MAGNETIC IMPURITIES}

\section{A. Abrikosov-Gor'kov model}

Now we turn to the situation when the BCS coherence peak is smeared by magnetic disorder. We restrict ourselves to the AG model [3] of Gaussian point-like magnetic impurities (a more general case will be considered elsewhere [35]) specified by the correlation function of the exchange field:

$$
\left\langle h_{i}(\mathbf{r}) h_{j}\left(\mathbf{r}^{\prime}\right)\right\rangle=\frac{\delta_{i j} \delta\left(\mathbf{r}-\mathbf{r}^{\prime}\right)}{6 \pi \nu \tau_{s}},
$$

where $\tau_{s}$ is the spin-flip scattering time. The latter plays the role of the pair-breaking time which determines the pair-breaking parameter

$$
\eta=\frac{1}{\tau_{s} \Delta_{0}} .
$$

The vector $\mathbf{h}$ is three-dimensional, while the effective dimensionality of the sample may be different.

Formation of the subgap states in a superconductor with weak magnetic impurities was studied by Lamacraft and Simons [13] who came to the result essentially coinciding with Eq. (7). However, inspired by the preceding analysis of the ROP model one may expect that there should be instantons related to optimal fluctuations of the exchange field $\mathbf{h}$ and/or order parameter field $\Delta(\mathbf{r})$, leading to the Larkin-Ovchinnikov tail (5). Below we study this instanton contribution and demonstrate that the main part of the subgap DOS tail may be described either by the Lamacraft-Simons or by the Larkin-Ovchinnikov results, depending of the values of $g_{\xi}$ and $\eta$ [see Sec. $\mathrm{VD}$. In the case when the tail is due to the Larkin-Ovchinnikov optimal fluctuation, it arises as a result of mesoscopic fluctuations of the order parameter. 


\section{B. Sigma-model action}

We use the real-energy replica sigma model introduced in Sec. IIB. Before averaging over magnetic disorder the initial action expanded to the second order in the impurity magnetization $\mathbf{h}(\mathbf{r})$ takes the form [23]:

$$
S=S_{0}+S_{1}+S_{2}
$$

where $S_{0}$ is the action for the uniform superconductor given by Eq. (14), and the terms $S_{1,2}$ describe magnetic impurities:

$$
\begin{gathered}
S_{1}=-i \pi \nu \int d \mathbf{r} \mathbf{h}(\mathbf{r}) \operatorname{tr}\left(\tau_{3} \boldsymbol{\sigma} Q\right) \\
S_{2}=-\frac{(\pi \nu)^{2}}{2} \int d \mathbf{r} h_{i}(\mathbf{r}) h_{j}\left(\mathbf{r}^{\prime}\right) \operatorname{tr}\left(\tau_{3} \sigma_{i} Q \tau_{3} \sigma_{j} Q\right),
\end{gathered}
$$

where $\sigma_{i}$ are Pauli matrices in the spin space.

Averaging over $\mathbf{h}$ with the correlation function (65) generates two terms, $S_{\eta}=\left\langle S_{2}\right\rangle$ and $S_{\text {dis }}=-\left\langle S_{1}^{2}\right\rangle / 2$, with different structures in the replica space:

$$
\begin{gathered}
S_{\eta}=-\frac{\pi \nu \Delta_{0} \eta}{12} \int d \mathbf{r} \operatorname{tr}\left(\tau_{3} \boldsymbol{\sigma} Q\right)^{2}, \\
S_{\mathrm{dis}}=\frac{\pi \nu \Delta_{0} \eta}{12} \int d \mathbf{r}\left(\operatorname{tr} \tau_{3} \boldsymbol{\sigma} Q\right)^{2},
\end{gathered}
$$

where the depairing parameter $\eta$ is given by Eq. (66).

As a result, the effective action describing gap fluctuations in the presence of a Gaussian short-range magnetic disorder takes the form:

$$
S=S_{0}+S_{\eta}+S_{\mathrm{dis}}
$$

The structure of the terms $S_{\eta}$ [Eq. (70)] and $S_{\text {dis }}$ [Eq. (71)] is pretty similar to that of the analogous terms, (16) and (15), in the ROP model. Note that contrary to the ROP model, the depairing term $S_{\eta}$ is generated automatically after averaging over $\delta$-correlated magnetic disorder.

In the analysis of the action (72), Lamacraft and Simons [13] considered only singlet configurations of the field $Q(\mathbf{r})$. Then the term $S_{\text {dis }}$ can be discarded, while the term $S_{\eta}$ just coincides with the analogous term (16) in the ROP model. In the absence of a field responsible for optimal fluctuations, the authors of Ref. [13] reproduced the result (7).

\section{Effective fluctuators in the singlet sector}

In order to go beyond the analysis of Ref. 13 one has to identify an effective fluctuator in the singlet sector which might be responsible for the Larkin-Ovchinnikov optimal fluctuation at $\varepsilon \ll \varepsilon_{*}(K \rightarrow \infty)$. We focus on the singlet sector since it becomes massless at $E \rightarrow E_{g}$, whereas the triplet is not [see Eqs. A3 and (A11)]. Therefore the instanton solution with the stretched-exponent action of the type (4) may arise only in the singlet component of $Q(\mathbf{r})$.

There are several sources of fluctuations in the singlet sector:

(i) Mesoscopic fluctuations of the order parameter with the correlation function given by Eq. (64). They arise due to fluctuations of potential impurities and are insensitive to weak magnetic disorder.

(ii) Fluctuations in the singlet component of the Green function $Q(\mathbf{r})$ generated through its triplet component due to nonlinearity of the Usadel equation (referred to as direct fluctuations). These fluctuations can be described in terms of an effective order parameter field $\Delta_{1}^{(\Phi)}(\mathbf{r})$. Its correlation function is calculated in Appendix. In the limit $T=0$ and $q=0$ it can be estimated as

$$
\frac{f^{(\Phi)}(0)}{\Delta_{0}^{2} \xi^{d}} \sim \frac{\eta^{(4+d) / 6}}{g_{\xi}^{2}} .
$$

(iii) Fluctuations of the order parameter due to randomness in $\mathbf{h}$ (referred to as indirect fluctuations) calculated in Appendix. In the limit $T=0$ and $q=0$ the corresponding correlation function can be estimated as

$$
\frac{f(0)}{\Delta_{0}^{2} \xi^{d}} \sim \frac{\eta^{2}}{g_{\xi}^{2}} .
$$

The presence of the the factors $g_{\xi}^{2}$ in the denominators of Eqs. (73) and (74) can be easily explained. Due to the vector structure of the random field $\mathbf{h}(\mathbf{r})$, an effective fluctuator in the singlet sector will be proportional to $\mathbf{h}^{2}$, with its variance, $f$, scaling as $\left\langle\mathbf{h}^{2}\right\rangle^{2} \propto \nu^{-2} \propto g_{\xi}^{-2}$.

\section{Result}

Comparing Eqs. (64), (73) and (744) we conclude that mesoscopic fluctuations of the order parameter is the leading source of disorder in $\Delta(\mathbf{r})$ for the magnetic impurities model. Therefore there is a competition of the Larkin-Ovchinnikov result (5) with $f(0)$ given by Eq. (64) and the Lamacraft-Simons dependence (7). According to Sec. IVA, the winner depends on the values of $g_{\xi}$ and $\eta$ :

- Regime I is realized for $g_{\xi}>\eta^{-\frac{2(8-d)}{3(4-d)}}$. The subgap DOS follows Eq. (7).

- Regime II is realized for $g_{\xi}<\eta^{-\frac{2(8-d)}{3(4-d)}}$. The subgap DOS follows Eq. (5).

\section{CONCLUSION}

This work was motivated by the discrepancy of the two instanton approaches to the problem of the subgap 
states in disordered superconductors. We have analyzed the replica structure of a generic instanton solution and demonstrated that the instanton of Larkin and Ovchinnikov 7] can be continuously deformed to the instanton of Simons and others [12, 13, 16] with decreasing the energy into the classically forbidden region.

Existence of two different instanton types is related to the presence of two types of disorder in the system: (i) the potential disorder responsible for diffusive motion of electrons and (ii) extra randomness in the some other characteristics of the sample, e.g., the Cooper coupling constant, the order parameter field, random spin exchange field, etc. In the quasiclassical theory of dirty superconductors, the potential (type-i) disorder is averaged out in the very beginning. The resulting Usadel equations are nonlinear already in the absence of type-ii disorder. Averaging over the latter brings an additional nonlinearity, which competes with the intrinsic nonlinearity of the problem. The relative strength of the two nonlinear terms is controlled by the proximity to the gap edge. For $\varepsilon \ll \varepsilon_{*}$, intrinsic nonlinearity is not important and the situation is similar to the problem of the linear Schrödinger equation with disorder. The instanton then corresponds to the optimal fluctuation of the random field, yielding the Larkin-Ovchinnikov result (5). In the opposite limit, $\varepsilon \gg \varepsilon_{*}$, only intrinsic nonlinearity of the problem is relevant. The nonlinear equations of motion still allow a bounce solution corresponding to the instanton of Simons and others.

Physically, the instanton of Larkin and Ovchinnikov describes an optimal fluctuation of the order parameter field which decreases the local value of the gap. The instanton of Simons and others describes mesoscopic fluctuations of quasiparticle response at a fixed value of the order parameter.

Depending on the parameters of the problem it might happen that the instanton action at the crossover energy, $\varepsilon \sim \varepsilon_{*}$, is smaller that 1 . In this situation, the LarkinOvchinnikov instanton does not exist and the density of the subgap states is described by Eq. (7). Otherwise the main part of the tail is described by Eq. (5), while its far asymptotics (77) is practically unobservable.

This general structure of the subgap DOS tail is analyzed for a number of superconducting problems with disorder. In particular, we reconsidered the gap smearing in the Abrikosov-Gor'kov model of weak paramagnetic impurities [13] and showed that depending on the parameters of the problem the DOS tail is described either by Eq. (5) or by Eq. (7).

Finally, we emphasize that our analysis applies to dirty superconductors and NS hybrids with diffusive electron dynamics described by the Usadel equation. Much less is known on the nature of the proximity gap in ballistic chaotic systems which is determined by the competition of the mean free time, the Ehrenfest time, and the escape time [36 38]. Going beyond the mean-field analysis and generalizing our findings to that type of systems remains an open problem.

\section{Acknowledgments}

We thank Ya. V. Fominov and S. E. Korshunov for useful discussions. This work was partially supported by the Russian Ministry of Education and Science (Contract No. 8678), the program "Quantum mesoscopic and disordered structures" of the RAS, and RFBR grant No. 13-02-01389.

\section{Appendix A: Disorder in the singlet sector due to magnetic impurities}

In this Appendix we calculate the correlation functions of effective disorder in the singlet sector due to randomness in $\mathbf{h}(\mathbf{r})$.

\section{Triplet Usadel equation}

Induced magnetization can be described with the help of the triplet Usadel equations. Following Ref. 39 we parametrize the $Q$ matrix in terms of the spectral angle $\theta$ and the magnetization vector $\mathbf{M}$ as

$$
\begin{aligned}
Q_{0}=M_{0} \sigma_{0}\left(\tau_{3} \cos \theta\right. & \left.+\tau_{1} \sin \theta\right) \\
& +i \mathbf{M} \boldsymbol{\sigma}\left(\tau_{3} \sin \theta-\tau_{1} \cos \theta\right),
\end{aligned}
$$

where $M_{0}=\sqrt{1+\mathbf{M}^{2}}$. The resulting equations for the singlet $(\theta)$ and triplet $(\mathbf{M})$ components in the Matsubara representation have the form [23]:

$$
\begin{array}{r}
\frac{D}{2} \nabla^{2} \theta+M_{0}\left(-\epsilon \sin \theta+\Delta_{0} \cos \theta\right)-(\mathbf{h} \mathbf{M}) \cos \theta \\
-\Delta_{0} \eta\left(1+\frac{2}{3} \mathbf{M}^{2}\right) \sin \theta \cos \theta=0, \\
\frac{D}{2}\left(\mathbf{M} \nabla^{2} M_{0}-M_{0} \nabla^{2} \mathbf{M}\right)+\mathbf{M}\left(\epsilon \cos \theta+\Delta_{0} \sin \theta\right) \\
-M_{0} \mathbf{h} \sin \theta_{0}+\frac{1}{3} \Delta_{0} \eta M_{0} \mathbf{M} \cos 2 \theta=0 .
\end{array}
$$

In the absence of $\mathbf{h}$, we have $\mathbf{M}=0$ and the spectral angle $\theta_{0}(\epsilon)$ should be obtained from the AG equation (22) analytically continued to Matsubara energies, $i E \rightarrow-\epsilon$.

The linear response of the magnetization $\mathbf{M}$ to the field $\mathbf{h}$ can be found from the triplet equation (A2b), which yields in the momentum representation:

$$
\mathbf{M}(\mathbf{q})=\frac{\sin \theta_{0}}{\xi^{2} q^{2}+\mu_{t}(\epsilon)} \frac{\mathbf{h}(\mathbf{q})}{\Delta_{0}},
$$

where

$$
\mu_{t}(\epsilon)=\frac{\epsilon}{\Delta_{0}} \cos \theta_{0}(\epsilon)+\sin \theta_{0}(\epsilon)+\frac{\eta}{3} \cos 2 \theta_{0}(\epsilon)
$$

has the meaning of a mass of the triplet modes. 
Now expanding the singlet equation (A2a) to the second order in $\mathbf{h}$ and using Eq. (22) we obtain

$$
-\xi^{2} \nabla^{2} \theta+\left(\epsilon / \Delta_{0}\right) \sin \theta-\cos \theta+\eta \sin \theta \cos \theta=\Phi(\mathbf{r}),
$$

where $\Phi_{\epsilon}(\mathbf{r})$ acts as an effective source of singlet fluctuations:

$$
\Phi_{\epsilon}(\mathbf{r})=-\frac{\eta \mathbf{M}^{2}}{6} \sin \theta_{0}(\epsilon) \cos \theta_{0}(\epsilon)-\frac{\mathbf{h M}}{\Delta_{0}} \cos \theta_{0}(\epsilon)
$$

A nonzero average $\left\langle\Phi_{\epsilon}(\mathbf{r})\right\rangle$ leads to renormalization of $\Delta_{0}$ and $\eta$, while the strength of disorder in the singlet sector is determined by the irreducible correlator

$$
\left\langle\left\langle\Phi_{\epsilon} \Phi_{\epsilon^{\prime}}\right\rangle\right\rangle_{\mathbf{q}}=\frac{\eta^{2} \sin 2 \theta_{0} \sin 2 \theta_{0}^{\prime}}{96\left(\pi \nu \Delta_{0}\right)^{2}} \int Z_{\epsilon}(k, q) Z_{\epsilon^{\prime}}(k, q) \frac{d^{d} \mathbf{k}}{(2 \pi)^{d}},
$$

where $\theta_{0}=\theta_{0}(\epsilon), \theta_{0}^{\prime}=\theta_{0}\left(\epsilon^{\prime}\right)$,

$Z_{\epsilon}(k, q)=\Pi_{\epsilon}(k)+\Pi_{\epsilon}(k-q)+\frac{\eta \sin ^{2} \theta_{0}(\epsilon)}{3} \Pi_{\epsilon}(k) \Pi_{\epsilon}(k-q)$,

and

$$
\Pi_{\epsilon}(q)=\frac{1}{\xi^{2} q^{2}+\mu_{t}(\epsilon)}
$$

is the triplet diffusion propagator on top of the AG state.

Further analysis goes differently for direct and indirect fluctuations of $\Phi(\mathbf{r})$.

\section{Direct fluctuations of $\Phi$}

According to Eq. A5 , behavior of quasiparticles with energy $\epsilon$ in the field of a fluctuating $\Phi_{\epsilon}(\mathbf{r})$ and constant $\Delta(\mathbf{r})=\Delta_{0}$ is formally equivalent to that in the field of a fluctuating order parameter with

$$
\Delta_{1}^{(\Phi)}(\mathbf{r})=\frac{\Delta_{0} \Phi_{\epsilon}(\mathbf{r})}{\cos \theta_{0}(\epsilon)}
$$

For the problem of the DOS tail we need real energies near the gap edge, $\epsilon=-i E \rightarrow-i E_{g}$. Since the triplet sector remains massive at the edge, we may simply evaluate $\Phi_{\varepsilon}$ right at $\epsilon=-i E_{g}$, when

$$
\mu_{t}\left(-i E_{g}\right) \equiv \xi^{2} r_{s}^{-2}=\frac{4}{3} \eta^{1 / 3}\left(1-\frac{1}{2} \eta^{2 / 3}\right)
$$

Here $r_{s}$ is the spin-rigidity length at the gap edge, which is finite in contrast to a divergent length $L_{E}$ in the singlet sector [Eq. (32)].

Fluctuations of the field $\Delta_{1}^{(\Phi)}(\mathbf{r})$ are characterized by the irreducible correlator $f^{(\Phi)}(\mathbf{q})=\left\langle\left\langle\Delta_{1}^{(\Phi)} \Delta_{1}^{(\Phi)}\right\rangle\right\rangle_{\mathbf{q}}$ which can be extracted from Eqs. (27), (A7) and (A10):

$$
f^{(\Phi)}(q)=\frac{\eta^{4 / 3}}{24(\pi \nu)^{2}} \int Z_{-i E_{g}}^{2}(k, q) \frac{d^{d} \mathbf{k}}{(2 \pi)^{d}},
$$

where in calculating $Z_{-i E_{g}}(k, q)$ it should be taken into account that $\sin \theta_{0}\left(-i E_{g}\right)=\cosh \psi_{g}=\eta^{-1 / 3}$. The correlation length of the field $\Delta_{1}^{(\Phi)}(\mathbf{r})$ is of the order of $r_{s}$, and the zero-momentum correlation function can be estimated as

$$
\frac{f^{(\Phi)}(0)}{\Delta_{0}^{2} \xi^{d}} \sim \frac{\eta^{4 / 3}}{g_{\xi}^{2}}\left(\frac{r_{s}}{\xi}\right)^{4-d}
$$

leading to Eq. (73).

\section{Indirect fluctuations of $\Phi$}

The field $\Phi_{\epsilon}(\mathbf{r})$ also affects quasiparticle behavior indirectly by inducing quenched inhomogeneity in the order parameter field. Fluctuations of $\Delta(\mathbf{r})$ can be obtained from the linearized self-consistency equation [compare with Eq. (56)]:

$$
\lambda^{-1} \Delta_{1}(\mathbf{q})=\pi T \sum_{\epsilon}\left[\frac{\partial F(\epsilon, \mathbf{q})}{\partial \Delta(\mathbf{q})} \Delta_{1}(\mathbf{q})+\delta F(\epsilon, \mathbf{q})\right]
$$

where $\delta F(\epsilon, \mathbf{q})=\delta \sin \theta(\epsilon, \mathbf{q})$ is a fluctuating part of the anomalous Matsubara Green function evaluated at a constant $\Delta(\mathbf{r})=\Delta_{0}$. Solving for $\Delta_{1}(\mathbf{q})$ we get

$$
\Delta_{1}(\mathbf{q})=L_{0}(q) \pi T \sum_{\epsilon} \delta F(\epsilon, \mathbf{q})
$$

where $L_{0}(q)$ is the static fluctuation propagator on top of the AG solution with a finite $\eta$ [note that Eq. (60) refers to the BCS case with $\eta=0$ ]. It is given by

$$
L_{0}^{-1}(q)=\frac{\pi T}{\Delta_{0}} \sum_{\epsilon}\left[\sin \theta_{0}(\epsilon)-\frac{\cos ^{2} \theta_{0}(\epsilon)}{\xi^{2} q^{2}+\mu_{s}(\epsilon)}\right],
$$

where $\mu_{s}(\epsilon)$ is the mass of the singlet modes [compare with Eq. (A4)]:

$$
\mu_{s}(\epsilon)=\frac{\epsilon}{\Delta_{0}} \cos \theta_{0}(\epsilon)+\sin \theta_{0}(\epsilon)+\eta \cos 2 \theta_{0}(\epsilon) .
$$

In the non-magnetic case $(\eta=0)$, Eq. (A16) reduces to Eq. (60).

The correction $\delta F(\epsilon, \mathbf{q})=\cos \theta_{0}(\epsilon) \delta \theta(\epsilon, \mathbf{q})$ induced by magnetic disorder follows from Eq. (A5):

$$
\delta F(\epsilon, \mathbf{q})=\frac{\cos \theta_{0}(\epsilon) \Phi_{\epsilon}(\mathbf{q})}{\xi^{2} q^{2}+\mu_{s}(\epsilon)} .
$$

For the irreducible correlator $f(\mathbf{q})=\left\langle\left\langle\Delta_{1} \Delta_{1}\right\rangle\right\rangle_{\mathbf{q}}$ we get:

$$
f(q)=L_{0}^{2}(q)(\pi T)^{2} \sum_{\epsilon, \epsilon^{\prime}} \frac{\cos \theta_{0}(\epsilon) \cos \theta_{0}\left(\epsilon^{\prime}\right)\left\langle\left\langle\Phi_{\epsilon} \Phi_{\epsilon^{\prime}}\right\rangle\right\rangle_{\mathbf{q}}}{\left[\xi^{2} q^{2}+\mu_{s}(\epsilon)\right]\left[\xi^{2} q^{2}+\mu_{s}\left(\epsilon^{\prime}\right)\right]}
$$

The field $\Delta_{1}(\mathbf{r})$ is correlated at the scale of the zerotemperature coherence length, $r_{c} \sim \xi(0)$, and its correlation function at $T=0$ and $q=0$ is given by Eq. (74). 
Note that in the limit $\eta \ll 1$ the correlation function of indirect fluctuations is much smaller than the correlation function of direct fluctuations [Eq. A13 ]. This is a consequence of the fact that $\Delta_{1}^{(\Phi)}$ accumulates fluc- tuations from the region of the size of the spin length $r_{s}$, whereas $\Delta_{1}$ accumulates fluctuations from the much smaller region of the size of the coherence length $\xi$.
[1] A. A. Abrikosov and L. P. Gor'kov, Zh. Eksp. Teor. Fiz. 35, 1558 (1958); 36, 319 (1959) [Sov. Phys. JETP 8, 1090 (1959); 9, 220 (1959)].

[2] P. W. Anderson, J. Phys. Chem. Solids 11, 26 (1959).

[3] A. A. Abrikosov and L. P. Gor'kov, Zh. Eksp. Teor. Fiz. 39, 1781 (1960) [Sov. Phys. JETP 12, 1243 (1961)].

[4] A. Anthore, H. Pothier, and D. Esteve, Phys. Rev. Lett. 90, 127001 (2003).

[5] K. Maki, Prog. Teor. Phys. (Kyoto) 29, 333 (1963); 31, 731 (1964).

[6] K. Maki in Superconductivity, edited by R. D. Parks (Marcel Dekker, New York, 1969), p. 1035.

[7] A. I. Larkin and Yu. N. Ovchinnikov, Zh. Eksp. Teor. Fiz. 61, 2147 (1971) [Sov. Phys. JETP 34, 1144 (1972)].

[8] J. Zittartz and J. S. Langer, Phys. Rev. 148, 741 (1966).

[9] I. M. Lifshitz, Zh. Eksp. Teor. Fiz. 53, 743 (1968) [Sov. Phys. JETP 26, 462 (1972)].

[10] K. Usadel, Phys. Rev. Lett. 25, 507 (1970).

[11] In Ref. 7 only the $3 \mathrm{D}$ case with weak disorder, $\eta \ll 1$, was considered, but generalization of this result to arbitrary dimensionality $d$ is straightforward.

[12] J. S. Meyer and B. D. Simons, Phys. Rev. B 64, 134516 (2001).

[13] A. Lamacraft and B. D. Simons, Phys. Rev. Lett. 85, 4783 (2000); Phys. Rev. B 64, 014514 (2001).

[14] M. G. Vavilov, P. W. Brouwer, V. Ambegaokar, and C. W. J. Beenakker, Phys. Rev. Lett. 86, 874 (2001).

[15] P. M. Ostrovsky, M. A. Skvortsov, M. V. Feigel'man, Phys. Rev. Lett. 87, 027002 (2001).

[16] F. M. Marchetti and B. D. Simons, J. Phys. A: Math. Gen. 35, 4201 (2002).

[17] C. A. Tracy and H. Widom, Commun. Math. Phys. 159, 151 (1994); 177, 727 (1996).

[18] The inequality (11) can be replaced by a weaker inequality $r_{c}<L_{E}$, where $L_{E}$ is given by Eq. (32).

[19] M. V. Feigel'man and M. A. Skvortsov, Phys. Rev. Lett. 109, 1470022 (2012)

[20] K. B. Efetov, Supersymmetry in Disorder and Chaos (Cambridge Univ. Press, Cambridge, 1996).

[21] A. M. Finkel'stein, in Soviet Scientific Reviews, edited by
I. M. Khalatnikov (Harwood Academic, London, 1990), Vol. 14.

[22] A. Altland, B. D. Simons, and D. Taras-Semchuk, Adv. Phys. 49, 321 (2000).

[23] D. A. Ivanov, Ya. V. Fominov, M. A. Skvortsov, and P. M. Ostrovsky, Phys. Rev. B 80, 134501 (2009).

[24] A. Silva and L. B. Ioffe, Phys. Rev. B 71, 104502 (2005).

[25] G. Parisi and N. Sourlas, Phys. Rev. Lett. 43, 744 (1979).

[26] A. A. Golubov, M. Yu. Kupriyanov, and E. Il'ichev, Rev. Mod. Phys. 76, 411 (2004).

[27] D. Taras-Semchuk and A. Altland, Phys. Rev. B 64, 014512 (2001).

[28] F. Zhou, P. Charlat, B. Spivak, and B. Pannetier, J. Low Temp. Phys. 110, 841 (1998).

[29] B. L. Altshuler, Pis'ma Zh. Eksp. Teor. Fiz. 41, 530 (1985) [Sov. Phys. JETP Lett. 41, 648 (1985)]; P. A. Lee and A. D. Stone, Phys. Rev. Lett. 55, 1622 (1985).

[30] B. L. Altshuler and B. Z. Spivak, Zh. Eksp. Theor. Fiz. 92, 607 (1987) [Sov. Phys JETP 65, 343 (1987)].

[31] M. Houzet and M. A. Skvortsov, Phys. Rev. B 77, 057002 (2008).

[32] Mesoscopic Phenomena in Solids, Modern Problems in Condensed Matter Sciences Vol. 30, edited by B. L. Altshuler, P. A. Lee, and R. A. Webb, (North-Holland, Austerdam, 1991).

[33] M. A. Skvortsov and M. V. Feigel'man, Phys. Rev. Lett. 95, 057002 (2005).

[34] A. M. Finkestein, Pis'ma Zh. Eksp. Teor. Fiz. 45, 37 (1987) [Sov. Phys. JETP Lett. 45, 46 (1987)]; Physica B 197, 636 (1994).

[35] Ya. V. Fominov and M. A. Skvortsov, in preparation.

[36] S. Pilgram, W. Belzig and C. Bruder, Phys. Rev. B 62, $12462(2000)$.

[37] D. Taras-Semchuk and A. Altland, Phys. Rev. B 64, 014512 (2001).

[38] M. G. Vavilov and A. I. Larkin, Phys. Rev. B 67, 115335 (2003).

[39] D. A. Ivanov and Ya. V. Fominov, Phys. Rev. B 73, 214524 (2006). 